\section{In step}

Among the first minerals to crystallize from basaltic magma are chromites and olivines, which may therefore be expected to record the magma's initial chemical composition. No so, warn P. A. H. Scowen et al. (Contrib. Mineral. Petrol. 107, 8-20; 1991) - studies of chromites from Kilauea Iki lava lake in Hawaii show that they remain in equilibrium with the evolving lava composition, even when entirely encased within olivine. The lava lake was created in 1959 during an eruption of Kilauea and its rapidly changing composition has been closely monitored since. The fact that the chromites' composition also changed in so short a time indicates that they must exchange ions with the lava quickly, even when not in direct contact with it.

\section{More from less}

It may not satisfy the homeopaths, who claim the power to detect substances that are completely absent, but P.G. Gillespie and A.J. Hudspeth (Proc. natn. Acad. Sci. U.S.A. 88, 2563-2567; 1991) have devised a technique for the recognition of femtogram quantities of proteins, minor constituents for example in a single cell. The total protein in a mixture - in one case the contents of a retinal rod outer segment - is covalently labelled with biotin, before or after gel electrophoresis. After blotting (with certain precautions that go beyond the use of coffee whitener to block non-specific binding) it is detected on film by a chemiluminescent reaction, catalysed by avidin-coupled alkaline phosphatase. Use of a similarly conjugated second antibody is another option. A range of applications suggests itself, as for instance the detection of foreign proteins expressed in Xenopus eggs.

\section{No two ways}

Two tribes of experimenters in the physics of fundamental forces could be unwitting antagonists, suggest $\mathrm{S}$. $\mathrm{K}$. Lamoreaux et al. (Europhys. Lett. 14, 503-505; 1991). Workers trying to find out if neutrons can turn freely into their antiparticles hope to constrain attempts to develop 'grand unified theories' of the strong and electroweak forces of matter, whereas those trying to find out whether gravity acts in different ways on matter and antimatter hope to learn something of the quantum description of gravity. In their note, which brings together authors from each camp, Lamoreaux et al. point out that any difference in the gravitational interactions of neutrons and antineutrons would give them different energy making their interconversion impossible to observe. But the observation of neutron-antineutron conversion would greatly limit the possible difference in their gravitational energy.

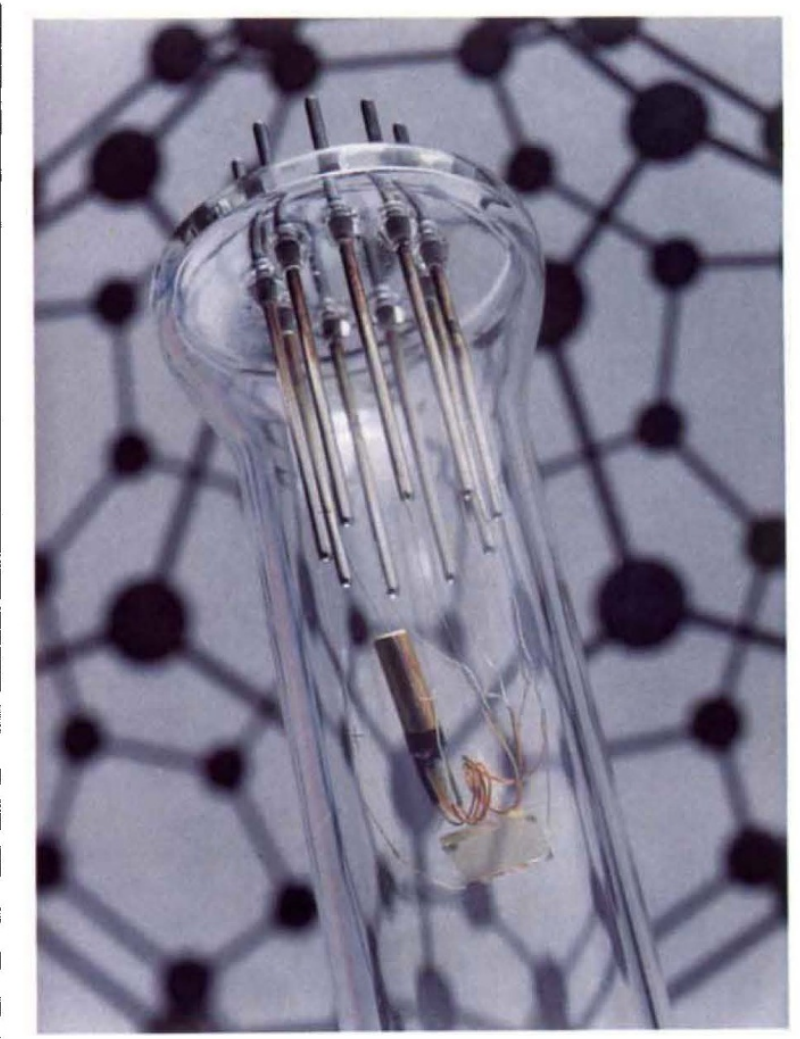

FIG. 2 The apparatus of Hebard et al. The superconducting doped $\mathrm{C}_{60}$ film is on the glass square near the bottom. The sealed tube contains inert helium to protect the fragile film.

terstitially between close-packed $\mathrm{C}_{60}$ spheres (Fig. 1). We can be relatively certain that the potassium has not found its way inside the pseudospheres. The potassium-doped $\mathrm{C}_{60}$ materials are highly reactive: the powders are pyrophoric, and the conducting films rapidly lose their conductivity on exposure to air. Greater stability might be possible if appropriate dopants could be placed inside the pseudospheres.

Another allotrope of carbon, graphite, can also be doped with potassium, through an intercalation reaction. In this case, however, the material is metallic both before and after intercalation. Intercalation of potassium into graphite also produces superconductivity ${ }^{6}$, but only at temperatures below $1 \mathrm{~K}$. In graphite, there is extended covalent bonding in two dimensions with weak Van der Waals bonding between the layers. For $\mathrm{C}_{60}$, there is covalent bonding within the pseudospheres but only weak Van der Waals bonding between pseudospheres. On doping $\mathrm{C}_{60}$, we may assume, potassium atoms lose an electron to the pseudospheres, partially populating a conduction band throughout the solid derived mainly from the 2 porbitals of carbon but with some contribution from the $2 s$ orbitals. These conduction electrons may well tend to bond the pseudospheres together, thus aiding in the electron transfer from pseudosphere to pseudosphere. There is every reason to believe that potassiumdoped $\mathrm{C}_{60}$ is isotropic. Some have felt that the two-dimensional character of the cuprate superconductors is a key factor for their high transition temperature. However, at least for carbon, three-dimensional bonding is apparently better for superconductivity than is twodimensional bonding.

Both $\mathrm{C}_{60}$ and $\mathrm{C}_{70}$ are properly regarded as new allotropes of carbon. However, they differ from the more familiar diamond and graphite allotropes in an important way. Both diamond and graphite are forms of carbon that are thermodynamically stable as solids over certain ranges of pressure and temperature. Molecules of $\mathrm{C}_{60}$ and $\mathrm{C}_{70}$ might well exist at thermodynamic equilibrium at very high temperatures in gas-phase carbon, but the solid forms of $\mathrm{C}_{60}$ and $\mathrm{C}_{70}$ are unlikely to be thermodynamically stable at any temperature or pressure. The point is important, because superconductivity is often associated with instabilities. Consistent with this association is the finding of 'high-temperature' superconductivity in the allotrope of carbon which is never stable as a solid. The copper and bismuth oxides that exhibit hightemperature superconductivity also appear to be metastable, at least at the temperatures at which they are superconducting ${ }^{7}$. Thus, ways of preparing new superconductors often involve tricks to defeat equilibrium. This can lead to challenging synthetic chemistry, as with $\mathrm{C}_{60}$ and $\mathrm{K}_{x} \mathrm{C}_{60}$.

Throughout the short history of superconductors, it was generally believed that highest transition temperatures would be found for transition-metal compounds. The discovery of superconductivity in perovskite oxides of bismuth $^{8,9}$ gave the first exception. $(\mathrm{Ba}, \mathrm{K}) \mathrm{BiO}_{3}$ now yields a transition temperature ${ }^{10}$ of $34 \mathrm{~K}$, and superconductivity persists up to $18 \mathrm{~K}$ in $\mathrm{K}_{x} \mathrm{C}_{60}$. Superconductivity is known for compounds based on the intermediate rows of post-transition metals, but the transition temperatures do not exceed $7 \mathrm{~K}$. Surely, there are more superconductors to be discovered in this region of the periodic table.

Arthur W. Sleight is in the Department of Chemistry at Oregon State University, Corvallis, Oregon 97331, USA.

1. Hebard, A. F. et al. Nature 350, 600-601 (1991).
2. Haddon, R. C. et al. Nature 350, 320-322 (1991).

2. Haddon, R. C. et al. Nature 350, 320-322 (1991).
3. Flemming, R. M. Proc. Mat. Res. Soc. Symp. G. Boston 1990 (in the press)

4. Heiney, P. A. et al. Phy. Rev. Letts (submitted).

5. Allemand, P.M. J. Am. chem. Soc. (in the press)

6. Hannay, N. B. et al. Phys. Rev. Letters 14, 225-226 (1965).

7. Sleight, A. W. Physica C 162-164, 3-7 (1989).

8. Sleight, A. W., Gillson, J. L. \& Bierstedt, P. E. Solid St. Commun. 17, 27-28 (1975).

9. Cava, R. J. et al. Nature 332, 814-816 (1988).

10. Jones, N. L. et al. J. Solid St. Chem. 78, 319-321 (1989). 